\title{
PRIMAGE project: predictive in silico multiscale analytics to support childhood cancer personalised evaluation empowered by imaging biomarkers
}

Luis Martí-Bonmatí ${ }^{*}$, Ángel Alberich-Bayarri ${ }^{2}$, Ruth Ladenstein ${ }^{3}$, Ignacio Blanquer ${ }^{4}$, J. Damian Segrelles ${ }^{4}$, Leonor Cerdá-Alberich ${ }^{5}$, Polyxeni Gkontra ${ }^{5}$, Barbara Hero ${ }^{6}$, J. M. García-Aznar ${ }^{7,8}$, Daniel Keim ${ }^{9}$, Wolfgang Jentner ${ }^{9}$, Karine Seymour ${ }^{10}$, Ana Jiménez-Pastor ${ }^{2}$, Ismael González-Valverde², Blanca Martínez de las Heras ${ }^{11}$, Samira Essiaf ${ }^{12}$, Dawn Walker ${ }^{13}$, Michel Rochette ${ }^{14}$, Marian Bubak ${ }^{15}$, Jordi Mestres ${ }^{16}$, Marco Viceconti ${ }^{17}$, Gracia Martí-Besa ${ }^{5}$, Adela Cañete ${ }^{11}$, Paul Richmond ${ }^{13}$, Kenneth Y. Wertheim ${ }^{13}$, Tomasz Gubala ${ }^{15}$, Marek Kasztelnik ${ }^{15}$, Jan Meizner ${ }^{15}$, Piotr Nowakowski ${ }^{15}$, Salvador Gilpérez ${ }^{18}$, Amelia Suárez ${ }^{18}$, Mario Aznar ${ }^{18}$, Giuliana Restante ${ }^{19}$ and Emanuele Neri ${ }^{19}$

\begin{abstract}
PRIMAGE is one of the largest and more ambitious research projects dealing with medical imaging, artificial intelligence and cancer treatment in children. It is a 4-year European Commission-financed project that has 16 European partners in the consortium, including the European Society for Paediatric Oncology, two imaging biobanks, and three prominent European paediatric oncology units. The project is constructed as an observational in silico study involving high-quality anonymised datasets (imaging, clinical, molecular, and genetics) for the training and validation of machine learning and multiscale algorithms. The open cloud-based platform will offer precise clinical assistance for phenotyping (diagnosis), treatment allocation (prediction), and patient endpoints (prognosis), based on the use of imaging biomarkers, tumour growth simulation, advanced visualisation of confidence scores, and machine-learning approaches. The decision support prototype will be constructed and validated on two paediatric cancers: neuroblastoma and diffuse intrinsic pontine glioma. External validation will be performed on data recruited from independent collaborative centres. Final results will be available for the scientific community at the end of the project, and ready for translation to other malignant solid tumours.
\end{abstract}

Keywords: Artificial intelligence, Biomarkers (tumour), Cloud computing, Diffuse intrinsic pontine glioma, Neuroblastoma

\footnotetext{
* Correspondence: marti_lui@gva.es

'Medical Imaging Department, La Fe University and Polytechnic Hospital \&

Biomedical Imaging Research Group (GIBI230) at La Fe University and

Polytechnic Hospital and Health Research Institute, Av. Fernando Abril

Martorell 106, 46026 Valencia, Spain

Full list of author information is available at the end of the article
}

\section{Springer Open}

(c) The Author(s). 2020 Open Access This article is licensed under a Creative Commons Attribution 4.0 International License, which permits use, sharing, adaptation, distribution and reproduction in any medium or format, as long as you give appropriate credit to the original author(s) and the source, provide a link to the Creative Commons licence, and indicate if changes were made. The images or other third party material in this article are included in the article's Creative Commons licence, unless indicated otherwise in a credit line to the material. If material is not included in the article's Creative Commons licence and your intended use is not permitted by statutory regulation or exceeds the permitted use, you will need to obtain permission directly from the copyright holder. To view a copy of this licence, visit http://creativecommons.org/licenses/by/4.0/. 


\section{Key points}

- An open-cloud platform for decision support in neuroblastoma and diffuse intrinsic pontine glioma is being developed.

- A decision support system guided by imaging and paediatric oncology experts under a user-centric approach will be developed.

- The platform will validate imaging biomarkers (computed tomography, magnetic resonance, positron emission tomography, ${ }^{131}$ I-metaiodobenzylguanidine imaging) and integrated data.

- The system will develop diagnostic multiscale models to predict disease progression.

\section{Background}

The digital transformation of healthcare systems has fostered innovative clinical workflows and quality improvements through value-based healthcare [1]. Nowadays, digital diagnosis tools (such as imaging, pathology, genomic analytics, wearable sensors) and patient electronic records (clinical profiling, treatment, endpoints) are key enabling factors for a new paradigm in routine clinical practice. This change is expected to promote clinical innovation models via real-world data-driven inferences revealing insights implicit in the data [2]. Real-world evidence can help answering existing questions and generating new knowledge in a more reproducible way [3].

Another key enabling factor to untap the enormous potential of in silico tools to assist in clinical healthcare is the current level of adoption of high-throughput screening techniques for diagnosis and disease progression monitoring. The amount of clinical, pathological, molecular and imaging data available is enormous. The possibility of integrating large volumes of highly heterogeneous data into in silico predictive tools has proven crucial to enhance model performance in various applicability domains [4].

Computational imaging allows the extraction of multiparametric data, leading to a new era in radiomics, characterised by high-throughput extraction, storage and analysis of a large amount of quantitative imaging features and parameters (imaging biomarkers) able to provide quantitative relevant information (virtual biopsies) for the early disease diagnosis, disease phenotyping, disease grading, targeting therapies, and evaluation of disease response to treatment [5].

The development of predictive models using computational algorithms and artificial intelligence, taking into account all types of clinical, pathology, molecular, and imaging information able to predict valid disease-related outcomes by learning from retrospective data, is a hot topic of scientific debate. The validity of these predictive models depends on the quantity, quality, and representativeness of the datasets used, being major limiting factors [6].

\section{Imaging biobanks and in silico models}

Oncologic imaging represents a suitable field for the discovery and validation of new biomarkers from different imaging modalities (such as computed tomography, magnetic resonance, positron emission tomography, and ultrasound), since cancer patients are frequently monitored for staging and treatment response follow-up [7]. Many imaging biomarkers have been proposed over the last years to measure tumour anatomy, morphology, pathophysiology, metabolism, or molecular profiles in order to estimate different cancer hallmarks, such as proliferation/ growth, angiogenesis, and evasion or metastasis [8]. However, very few biomarkers have so far entered routine clinical practice to guide clinical decisions $[9,10]$. The majority of oncology imaging biomarkers still require external validation at different centres before they can be properly qualified as robust and reproducible.

Mathematical and computational modelling of biological processes can be used to enhance quantitative understanding of biomedical phenomena, such as cancer progression [11], potentially incorporating patient-specific data to enrich the scope of therapeutic target identification. Models can describe the growth of solid tumours using discrete or continuous representations, with or without accounting for stochasticity [12]. PRIMAGE (predictive in silico multiscale analytics to support cancer personalised diagnosis and prognosis, empowered by imaging biomarkers) is a funded Horizon 2020 project (RIA, topic SC1-DTH-07-2018) where a combination of these approaches ensures the best of both worlds.

The PRIMAGE project focuses on the further development of in silico tools for a more personalised clinical management of childhood cancer by targeting clinical endpoints (CEPs), considering the progression of the growth of the tumour post-diagnosis, but not including the initial oncogenic processes active during embryogenesis. The project will utilise novel high-performance computing (HPC) approaches to provide computationally efficient and large scale in silico models resulting in a decision support system (DSS) which will hopefully provide improved health outcomes (Fig. 1).

\section{Neuroblastoma and diffuse intrinsic pontine glioma (DIPG)}

Data infrastructure, imaging biomarkers and models for in silico medicine research will be developed and validated in the context of neuroblastoma (NB) and diffuse intrinsic pontine glioma (DIPG).

NB is the most frequent solid cancer of the early childhood [13], and the diagnosis age has proven to be a crucial factor in its prognosis [14]. A number of risk factors have been identified and are already in use by the International Neuroblastoma Risk Group (INRG) [15, 16]. Major European groups involved in PRIMAGE have 


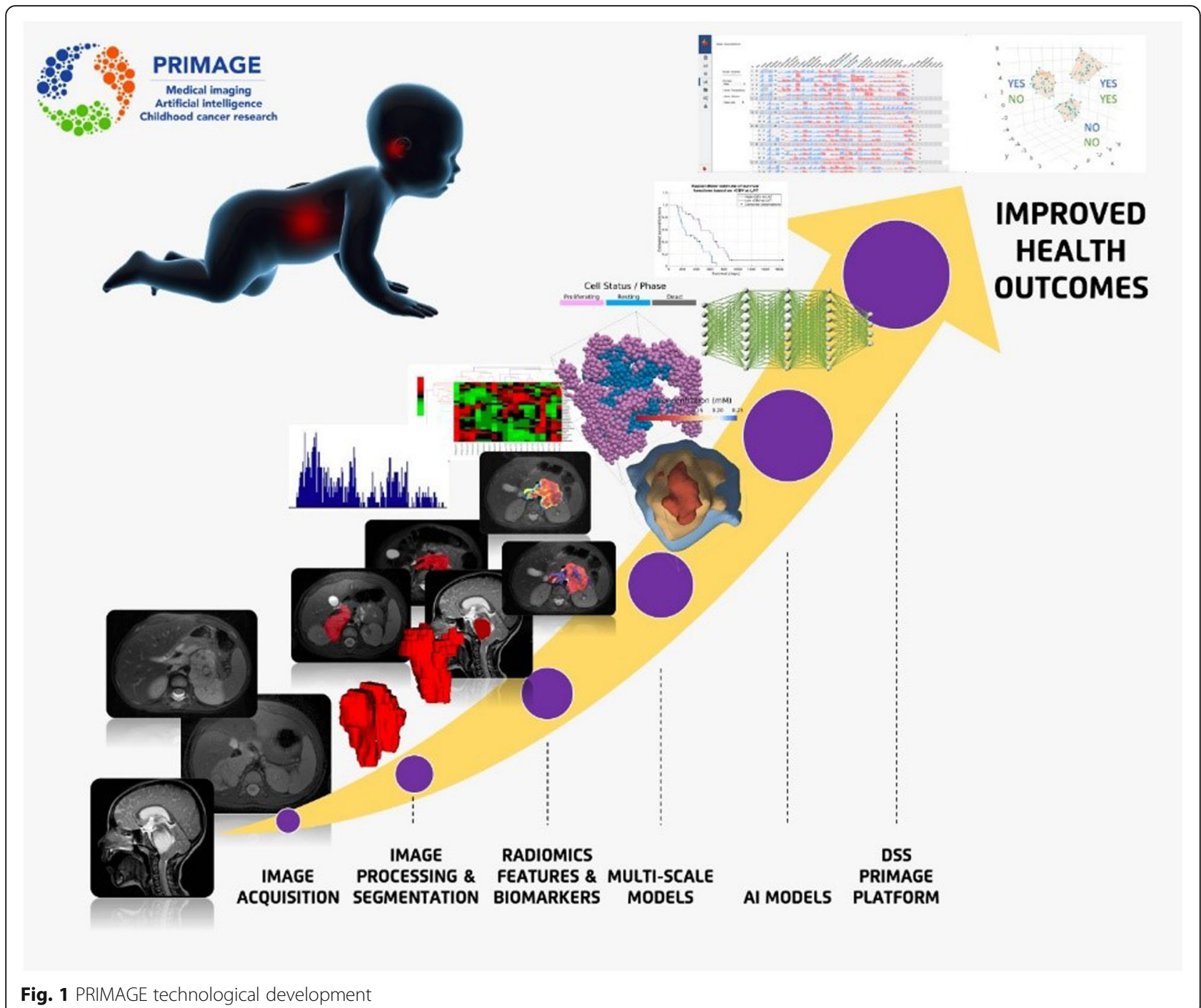

advanced standard of care treatments in low [17-19], intermediate [20, 21] and high-risk NB [22-24].

DIPG is the leading cause of brain tumour-related death in children [25]. Given the rarity of childhood tumours, international cooperative networks are essential to agglutinate relevant retrospective data and/or prospective cases for clinical trials, facilitating identification of effective tools for earlier diagnosis and potentially effective therapeutics.

The aim of the project is the development of an open hybrid cloud and HPC platform with later implementation and validation in non-interventional trials, which will support decision-making in the clinical management of malignant solid tumours. The PRIMAGE platform will implement the latest advancement of in silico computational image analysis and modelling which may be run on central processing unit (CPU) or general purpose graphics processing unit resources as needed.
The results are expected to have great impact not only on NB and DIPG but also on the management of other malignant solid tumours, since the proposed methodologies for data management, in silico models and visualisation tools will be available to be transferred to other cancer types.

The development process of the PRIMAGE platform following a user-centric approach is summarised in Fig. 2.

\section{Methods}

The partnership

For the successful design and implementation of PRIMAGE, a very high level of interdisciplinarity consortium was required, with expertise ranging from HPC infrastructures to visual analytics [26] and multiscale simulation, bringing together public and private organisations across Europe to perform collaborative research and development, a key aspect of the PRIMAGE interdisciplinary approach. Partners from eight European countries were selected (hospitals, research and 


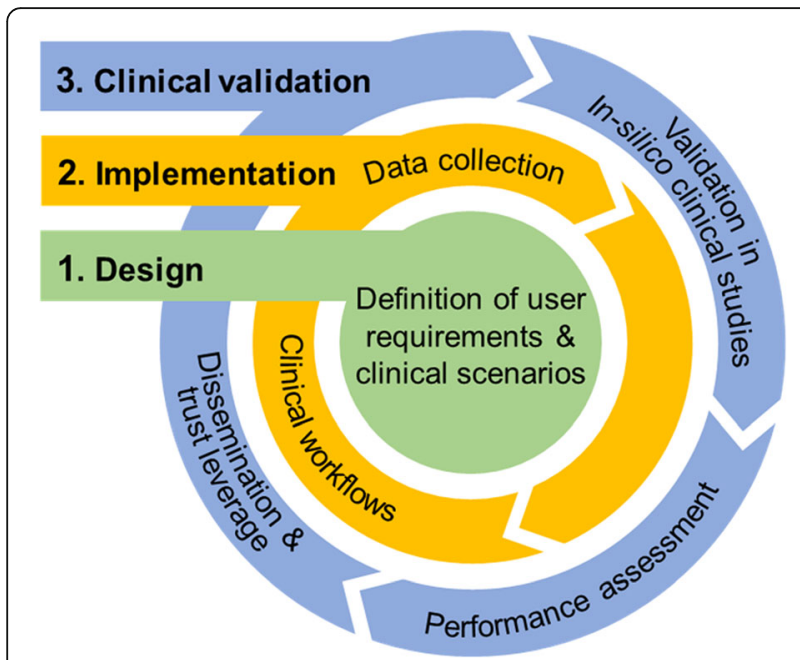

Fig. 2 PRIMAGE approach

development centres, medical associations, private companies, and universities), constituting a pan-European ecosystem of knowledge, infrastructures, biobanks, and technologies in the area of oncology, in silico, and cloud computing/HPC healthcare-related computing. Following a recruitment process, 16 organisations were incorporated, all of them are well known and with remarkable expertise in their respective areas. The University and Polytechnic Hospital La Fe in Spain is the project coordinator institution.

On the clinical side, the PRIMAGE consortium has tried to reunite leading European clinical centres together with key opinion leaders specialised in NB and DIPG (as these cancers are the context of the application proposed for the validation works of PRIMAGE in silico tools during this project) (Table 1). The University and Polytechnic Hospital La Fe in Spain, the Children's
Cancer Research Institute in Austria, the University Hospital Cologne in Germany, and the Pisa University Hospital in Italy constitute the clinical team. All of them belong to the European Research Network in NB and DIPG, facilitating PRIMAGE access to their datasets for further secondary used studies, in addition to their own biobanks and databanks of existing DIPG and NB cases. Moreover, the European Society for Paediatric Oncology (SIOPE) joined PRIMAGE, leading its dissemination and communication activities.

The knowledge and expertise of universities, research centres and private companies are essential for successful development of PRIMAGE. Partners include Quantitative Imaging Biomarkers in Medicine (QUIBIM SME), Institute for Molecular Imaging Technologies and Mechanical Engineering Department at Valencia Polytechnical University, Chemotargets SME, and Matical Innovation in Spain; the Department of Computer Science at University of Konstanz in Germany; Medical Imaging Technologies (Medexpim) in France; the University of Sheffield in United Kingdom; Simulation, Modelling and Engineering software (Ansys group) in France; Akademia Gorniczo-Hutnicza Im (Cyfronet) in Poland; and the Department of Industrial Engineering at the University of Bologna in Italy.

The consortium partners ensure complementarity and bring the necessary combination of skill, knowledge, technology, and motivation, comprising a highly motivated team, fully committed to turning PRIMAGE into a case study in the use of existing datasets, serviceoriented architectures and in silico technologies for better diagnosis and treatment of oncology diseases.

An advisory board consisting of a recognised group of experts in the fields of paediatric oncology, imaging biomarkers and related information and communication technologies, General Data Protection Regulation and

Table 1 Clinical centres and networks data registries involved in data collection with an estimation of cases for neuroblastoma (NB) and diffuse intrinsic pontine glioma (DIPG)

\begin{tabular}{|c|c|}
\hline Responsible entity & Characteristics \\
\hline $\begin{array}{l}\text { Clinical partners for NB and DIPG } \\
\text { University and Polytechnic Hospital La Fe, Spain } \\
\text { Children's Cancer Research Institute, Austria } \\
\text { University Hospital Cologne, Germany } \\
\text { Pisa University Hospital, Italy }\end{array}$ & $\begin{array}{l}\text { Target sample: approximately } 900 \text { cases with imaging, clinical, and molecular data. } \\
\text { Data type: imaging (magnetic resonance, computed tomography, }{ }^{131} \text { I-metaiodine-benzylguanidine } \\
\text { scintigraphy and single-photon emission tomography, positron emission tomography/computed } \\
\text { tomography), histology (if available), complete molecular biology studies according to SIOPE } \\
\text { (blood, urine, and bone marrow, cerebrospinal fluid), genetic (next generation sequencing, } \\
\text { fluorescence in situ hybridisation), and clinical data (patient profile, prescribed treatment, survival). }\end{array}$ \\
\hline $\begin{array}{l}\text { Data on patients with NB } \\
\text { GPOH }\end{array}$ & $\begin{array}{l}\text { Target sample: approximately 1,000 NB (high, low, and intermediate risk) patients participants in } \\
\text { academia-promoted clinical trials. } \\
\text { Data type: diagnosis and longitudinal data (clinical, follow-up, and biology data for all patients } \\
\text { registered in GPOH database). }\end{array}$ \\
\hline $\begin{array}{l}\text { Data on patients with DIPG } \\
\text { SIOPE registry }\end{array}$ & $\begin{array}{l}\text { Target sample: approximately } 700 \text { DIPG patients from European Union countries, both inside } \\
\text { and outside clinical trials. } \\
\text { Data type: diagnostic and follow-up magnetic resonance scans linked to e-data transmittal form } \\
\text { including demographics, medical history, and physical exam at time of diagnosis, results from } \\
\text { radiological, results from pathological review (if available), treatment (including radiotherapy, } \\
\text { chemotherapy, surgery and supportive), clinical data, and last known status of the patient. }\end{array}$ \\
\hline
\end{tabular}


industry representatives of manufacturers of drugs and picture archiving and communication systems has been designated to give general advice and guidance to the consortium.

\section{Platform architecture}

The PRIMAGE in silico models to be developed require significant computational and data storage resources to process. Our intention is to deliver a bespoke information technology solution, combining large-scale HPC and versatile cloud computing resources for optimum efficiency and reliability.

The infrastructure will be ultimately based on a DSS which will be designed for cancer management with advanced functionality and usability under a user-centric approach, guided by the clinical partners. A diagram of a high-level architecture can be appreciated in Fig. 3. This DSS will make use of the following:

- Large-scale processing on HPC resources (CPU or general purpose graphics processing unit), overlaid by a convenient representational state transfer-based process controller called Rimrock [27] and data access suite called Polish Grid Infrastructure (PL-grid) Data [28].

- Hybrid cloud resources, composed of both private and public cloud sites (based on the Europen open science cloud, EOSC, services), which will host PRIMAGE data repositories. Computational tasks can be deployed and coherently managed by a single access tool, such as the Atmosphere platform (project number 777154, European Commission) [29].

- An integration middleware, consisting of a set of protocols and interfaces between HPC/storage (models), private and public cloud computing/ storage (repositories, sandboxed processing), and external data sources (anonymised clinical and biobanking data). The middleware will be put in place to achieve an adequate level of solution coherency.

- Upper layer service exposing the features of the underlying infrastructure to researchers as a convenient graphical user interface, to manage definition, execution, and comparison of results of computationally intensive modelling pipelines. The tool will feature security management and application programming interface for programmatic access, and it will be based on the model execution environment (MEE) [30], which has been developed and successfully deployed in the EurValve project [31-33].

\section{Data repositories}

Stored clinical data (imaging, clinical, pathology and molecular) from 2002 onwards will be collected by the clinical centres and the paediatric oncological associations involved in PRIMAGE project. Clinical, molecular and imaging data in PRIMAGE will be undertaken under the strictest administrative and contractual procedures to ensure legal and ethical compliance under the General Data Protection Regulation in Europe. All cases included within the data repository, both for model development and platform validation, will have to be approved by the Ethics Committees of their respective centres. Even more, the observational in silico trial design for the internal platform validation, including new data from 2020 to 2022 , will be constructed in parallel to standard clinical practice after signed consent is obtained.

Relevant data will be used following extraction, anonymisation and curation for computational simulation developments and testing the integrated PRIMAGE DSS platform. The datasets used for the training and the development of the different in silico models will differ from the datasets used for the later validation of the integrated platform. Therefore, the use of clinical data (in the big data domain) for model training and model testing will be done in two phases:

- Phase 1: compilation of clinical, molecular and imaging data for PRIMAGE training, knowledge extraction, and multiscale testing of the in silico models for tumour growth, advanced visualisation solutions, identification and analysis of imaging biomarkers and training of predictive models for CEPs.

- Phase 2: extensive in silico testing of the integrated PRIMAGE DSS platform will be undertaken using cases from the same retrospective dataset, split by cross validation from the curated initial clinical dataset to ensure that these specific datasets are not used in the training phase.

Big data techniques will be applied to generate new knowledge from the advanced in silico tools. The use of retrospective data for training models, testing and validation will require extensive curation and quality control procedures. Automated tools will be implemented in this project to streamline the processes of extracting, mapping data, controlling the quality and homogenisation, translation, and completion of data feeds. Allocation of significant human resources is also foreseen, as human intervention is essential to achieve excellence in the training data sets.

Clinical data used in the international staging and stratifying criteria for NB and DIPG, including age, disease extension, image defined risk factors, histological type, grade of tumour differentiation, genetic, and molecular features will be used at the models construction phase. Imaging biomarkers will be extracted and validated for their use in cancer management, in 


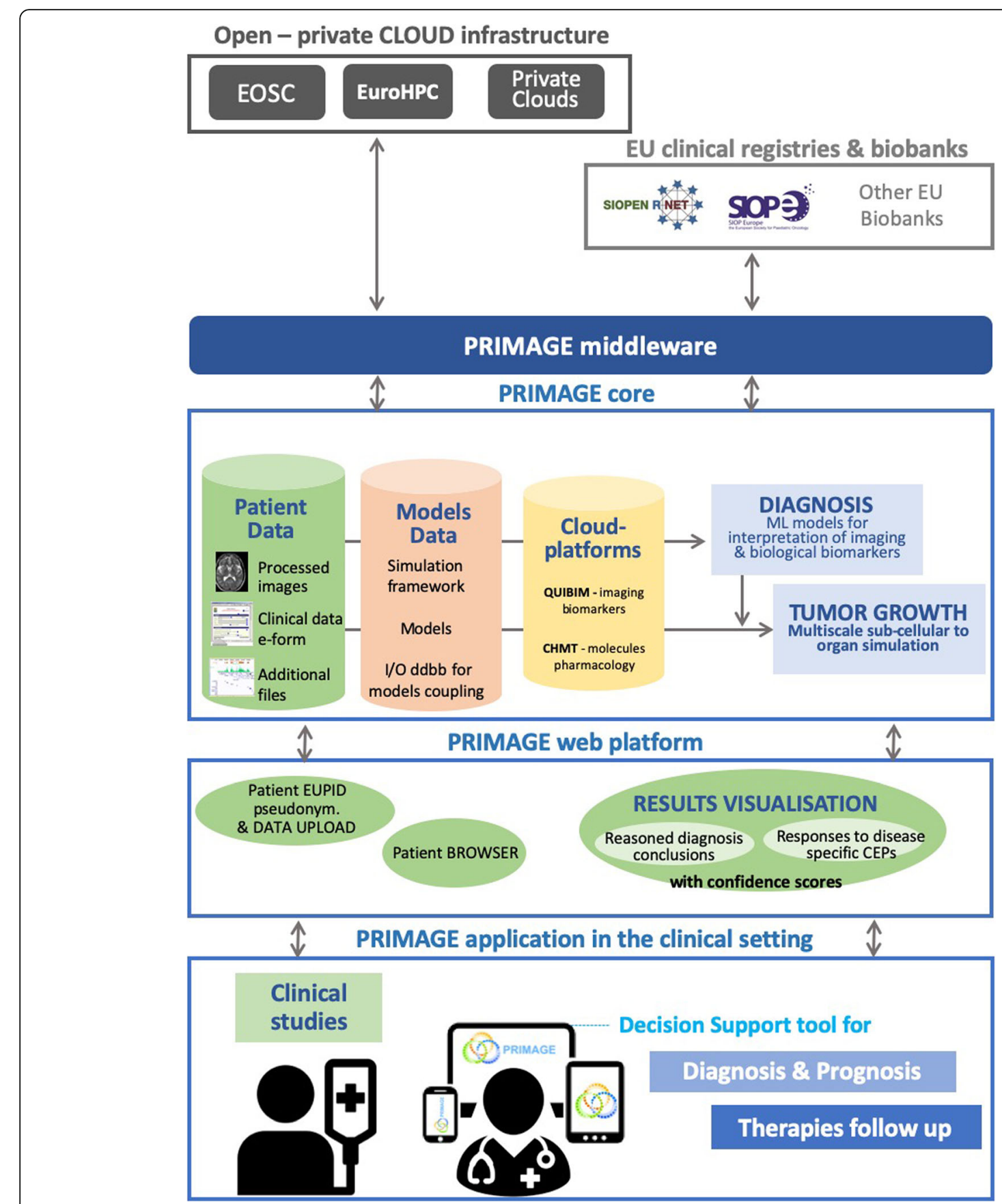

Fig. 3 PRIMAGE platform concept diagram

combination with already available biological biomarker panels. PRIMAGE is defined as an observational in silico study that will be validated also in silico in data recorded from new clinical observations. Image radiomics and dynamic parameters will be obtained from standard of care real-world ultrasound, computed tomography, magnetic resonance, positron emission tomography, and ${ }^{123} \mathrm{I}-$ metaiodobenzylguanidine scintigraphy or single-photon emission computed tomography images.
Experimental and computational methodologies will be used for the identification and validation of novel imaging biomarkers and the development of shared instruments for knowledge extraction from imaging biomarkers, clearly focused on improving disease diagnosis and follow-up. The development of a biomarker involves defining its relationship with the objective reality (structural, physiological, biological, or molecular), monitoring its technical validity and the relationship with the final CEPs. The path 
to handcrafted biomarker development, expansion and subsequent implementation is based on available guidelines and recommendations [34]. Main outcomes to be predicted relate to tumour phenotyping, treatment allocation, response to treatment and children survival.

Machine learning and image processing deep learning algorithms extract pattern information from the images and link outcome results to known ground-truth diagnosis. For this task, QUIBIM, a start-up company working in the field of artificial intelligence and imaging biomarkers applied to radiology data, will provide the methodologies for identification and validation of imaging biomarkers and related algorithms for the automatic analysis of images towards their validation in clinical trials through a PRIMAGE-specific customisation of QUIBIM precision platform (SME Instrument Phase 2, project number 778064). The computational equationbased handcrafted methods will be used first to have a ground truth for the training of machine learning algorithms. On a furthermore advanced stage, at the end of the project, it is envisioned that deep learning tools will directly provide clinical estimations from the source images, clinical and molecular data information.

\section{In silico scale models}

PRIMAGE's proposed in silico model spans three scales:

- The tumour scale model describes the evolution of volume and cellularity in the primary tumour, as well as its biomechanical interactions with the surrounding tissues, and the diffusion of nutrients and metabolites to and from the closest blood vessels. These quantities are described as spatial fields, whose temporal evolution is governed by a system of partial differential equations. These equations are discretised and then integrated over a period of time using a finite element method. Reduced order techniques will be applied on tumour parameters (variability of tumour size, material properties and treatment type) to enable real-time simulation at the tumour scale.

- The tissue scale model requires two complementary methodologies-firstly, a continuous, partial differential equation-based model that includes the chemical, biological and biomechanical interactions of the NB/DIPG cells with each other and with their extracellular matrix and vasculature. Model outputs will be the population sizes of different cell types and their evolution over time. In parallel to this, we will develop an agent-based model [35], wherein each cell is represented as an individual within a region of interest, explicitly capturing cellular behaviours including cell cycle progression, cell cycle arrest, cell death, production and degradation of the extracellular matrix, physical intercellular interactions, and cell-microenvironment interactions. This model may be used independently to explore hypotheses about specific regions of the tumour at a high resolution, or be fully integrated with other PRIMAGE models using state-of-the-art optimisation and acceleration techniques [36] and multiscale modelling approaches, as described below.

- The cell scale model describes the evolution of chemical and biological properties over time inside a single NB/DIPG cell, when it is exposed to different environmental conditions including various treatments.

\section{Computational strategy}

This project proposes a dataflow strategy to enable the proposed multiscale model to be executed using available HPC resources, in an effective and robust manner, considering that the proposed tumour model will need to be coupled to hundreds of thousands of tissue models, each coupled to also hundreds of thousands cell models.

In the proposed dataflow strategy, each single-scale model is described as a black box that takes as input an array of input sets and produces as output an array of output sets. Bi-directional resampling modules are proposed between the database that contains the input sets as computed, and those as required by the next model. Thus, resampling modules are used on value sets that are computed at lower scales and homogenised at upper scales, and on value sets that are computed at upper scales and then particularised at lower scales, at each scale transition.

The proposed workflow for the implementation of the described dataflow approach is:

- Implementation of the software infrastructure to manage the multiple databases. This infrastructure includes frameworks for coupling of the models at different scales via resampling functions, and repositories for the three single-scale models as they develop.

- Models execution without resampling. At this stage, no resampling will be provided as the software developments are focused on delivery and validation of the dataflow architecture to execute the multiscale model under suboptimal conditions, as efficiently as possible (using acceleration techniques).

- Models execution with resampling. Tissue-to-organ and cell-to-tissue modules will be incorporated in the dataflow, using progressively sophisticated multidimensional sampling techniques.

- Testing alternative approaches for enhancing computational efficiency. The use of surrogate modelling methods such as Gaussian processes will be explored as an alternative of resampling techniques. 
Although operating under completely different principles, they can be used to produce same result.

Even more, deep learning solutions will be developed, tested and validated for image segmentation (convolutional neural network architectures), radiomics analysis (support vector machine, linear discriminant, quadratic discriminant, decision trees, logistic regression, nearest neighbour, deep neural networks, and their combination in ensemble models) and CEPs (e.g., tumour subtypes, patient's prognosis), to estimate most accurate lesion diagnosis, treatment prediction, and patient prognosis. Models will be adapted to each application context (NB, DIPG) in order to more specifically address the clinical problems.

\section{Performance platform validation}

The proposed in silico tools will help tackle the relevant CEPs on the clinical target applications. The following CEPs have been prioritised by the Consortium clinicians as highly relevant to these diseases and suitable for being supported by in silico models, contributing to enhance reliability of current diagnosis and prognosis procedures:

- Prognosis of NB spontaneous regression capacity for low and intermediary risk patients;

- Identification of high-risk NB patients with imminent risk of relapse (50\% currently);

- Identification of NB high-risk patients that will not respond to induction chemotherapy (30\% currently);

- Identification of DIPG patients who will respond to treatment (10\% currently);

- Estimation of the expected survival period for DIPG responder patients.

A functional version of the PRIMAGE platform will be extensively evaluated in order to assess the platform's performance, thus its capacity to guide clinical decisions in a precise, reliable, and relevant manner. The datasets used for the validations includes imaging data, clinical data and genetics and other molecular data (Table 2).

The internal validation of PRIMAGE platform will be performed using datasets provided by the clinical centres and organisations involved in PRIMAGE project which never were used for the models and platform development.

In addition, in order to guarantee its correct performance under general conditions when using any datasets from the real world, PRIMAGE platform will be externally validated using data from clinical centres out of PRIMAGE environment. To get access to this data, other hospitals not involved in the project as partners have already been invited to participate by providing NB and DIPG cases to the PRIMAGE platform as independent collaborative international centres.
Table 2 Datasets used for testing of the PRIMAGE platform

\begin{tabular}{ll}
\hline Imaging data & Imaging data represents the highest challenge \\
& in terms of storage and processing. In \\
& PRIMAGE data repositories, for each patient, \\
& imaging data is linkable to their available \\
& pseudonymised biological, pathological, and \\
& genetics. The use of common metadata \\
& frameworks and image analysis techniques for \\
& automated data annotation for each image is \\
& proposed to generate common repositories \\
Genetics and other & This project uses existing knowledge on \\
molecular data & biological biomarkers (currently on clinical use \\
& or at advanced clinical validation stage). This \\
& type of data is used in combination with \\
& imaging and clinical data, facilitating \\
& multidisciplinary big data analytics. \\
Clinical data & Use of natural language processing tools for \\
automated extraction of relevant pathological \\
data, including data on patient response to \\
specific treatment will be extracted from the \\
electronic health record. Data will then be \\
structured, curated, and stored.
\end{tabular}

Table 3 describes the methodologies that will be used in the evaluation of PRIMAGE platform performance. During the evaluations, it will be also assessed other metrics such as security, reproducibility, interoperability and usability amongst others (Table 4).

\section{Expected results}

The state of the art for production-quality hybrid computational cloud and HPC for in silico processing of clinical cases is currently represented by projects such as EurValve [30, 31, 37] and GoSmart [38, 39], having achieved significant progress in developing integrated, comprehensive frameworks. EurValve has also come up with an integrated cloud/HPC computing solution to back up its MEE, dedicated for simulations of valvular heart conditions. This environment is now used in EurValve to perform clinical validation of the resulting DSS, which is a preliminary step towards development of an integrated, non-distributed clinical DSS.

The significant novelty of PRIMAGE with regard to this state of the art is to bring the cloud and HPC computing solutions, already successfully utilised for development and validation of in silico models, considerably closer to clinical use. The project will carefully evaluate available strategies, and it will deploy the selected solution to remotely provision the computationally intensive elements of the proposed clinical DSS, thus enabling the advantages of in-cloud computation for today's DSSs. The PRIMAGE approach combines the training and validation of models for medical imaging biomarkers and tumour growth simulation on open scientific cloud infrastructures, which constitutes the most computing intensive part, with the use of those models for personalised diagnosis, prognosis and optimisation of 
Table 3 PRIMAGE platform testing methodologies and performance metrics

\begin{tabular}{ll}
\hline Main testing methodologies \\
\hline infrastructure & $\begin{array}{l}\text { Definition of unitary and integration tests based on the } \\
\text { application requirements, monitoring along time, design-time } \\
\text { vulnerability analysis. }\end{array}$
\end{tabular}
Main performance metric

Performance (deployment and reconfiguration overheads, performance penalties, scalability), reliability (correct results with respect references), reproducibility (predictability of performance and automation), robustness (reliability along time and under different stress conditions), security (identification of vulnerabilities and isolation), privacy (privacy risk estimation).

High-performance Continuous monitoring of infrastructure. Alerts for computing administrators in case of malfunctions or failures. infrastructure Data repositories

Testing on MR, ${ }^{131}$ I-MBIG imaging, CT, PET/CT data, from retrospective studies of neuroblastoma and diffuse intrinsic pontine glioma patients

Imaging biomarkers

Testing on images (MR, ${ }^{131}$ I-MBIG imaging, CT, PET/CT) from retrospective data of neuroblastoma and diffuse intrinsic pontine patients

Multiscale modelling framework
Qualitative and quantitative comparison of numerical predictions with retrospective data
VM start-up time. Resource consumption. Number of concurrently running computational tasks. Availability. Measured through monitoring statistics, experiments and benchmarks.

Correlation between clusters of imaging biomarkers. Correlation between radiomic signatures and genomic profiles, and/or circulating tumour biomarkers from liquid biopsy (circulating tumour cells, tumour nucleic acids, etc.)

Precision, accuracy and clinical relationship measured in terms of quantified limit of detection and limit of quantification, reproducibility, sensitivity/specificity, coefficient of variation, correlation to diagnosis/prognosis of a specific disease

Quantitative correlation of the shape and size of tumour between image-based data and computer-based results. Qualitative correlation of vascular level and extracellular matrix properties in the tumour surroundings.

CT Computed tomography, MR Magnetic resonance, MBIG Metaiodobenzylguanidine, PET Positron emission tomography

treatment, within hospital boundaries. The construction of in silico clinical trials will allow decision-making from causal inference from observational databases emulating a pragmatic target trial if methodological pitfalls are avoided [2].

This project will bring major advancements in the validation of novel imaging biomarkers; it will create advanced computational models for tumour growth simulation, given response to specific CEPs. A very limited number of imaging biomarkers have been used in routine clinical practice to guide clinical decisions [7-9].
Therefore, PRIMAGE predictive models will enable unprecedented effectiveness in the translation from clinical Big Data to personalised predictors for malignant solid tumours, particularly NB and DIPG, by incorporating these assets for Big Data to usable clinical knowledge translation. The PRIMAGE diagnosis guiding tool uses quantification of imaging findings to improve sensitivity, specificity, accuracy and reproducibility of diagnostic and therapeutic decisions derived from image features used in combination with clinically validated biological biomarkers and cross-cohort machine learning from

Table 4 Metrics assessed in PRIMAGE platform

\begin{tabular}{|c|c|}
\hline Metrics to be assessed & Methodology \\
\hline Security/privacy & $\begin{array}{l}\text { Provision of authentication and authorisation and analysis of vulnerabilities from public databases. Assessment of the } \\
\text { platform's robustness to preserve data integrity according to GDPR, evaluating the privacy risk (e.g., as the capability of a } \\
\text { model to infer information previously anonymised), and managing the fine-grain consent as GDPR requires. }\end{array}$ \\
\hline Correctness/reliability & $\begin{array}{l}\text { Assess the correctness of the predictive results for the established clinical end points using testing datasets from clinical } \\
\text { data repositories for over } 2000 \text { neuroblastoma patients and over } 500 \text { diffuse intrinsic pontine glioma patients with } \\
\text { complete diagnosis and follow-up data, including treatment and outcomes. }\end{array}$ \\
\hline $\begin{array}{l}\text { Sensitiveness to } \\
\text { incomplete data }\end{array}$ & $\begin{array}{l}\text { Assess dependence of correctness of the predictive results with the completeness of the diagnosis datasets, to establish } \\
\text { how new biomarkers modify minimum datasets required for correct diagnosis/prognosis. }\end{array}$ \\
\hline Reproducibility & $\begin{array}{l}\text { Statistical assessments: dispersion in the results obtained for a subgroup of patients with a common clinical diagnosis, } \\
\text { belonging to different hospitals where the diagnosis studies were undertaken }\end{array}$ \\
\hline Interoperability & $\begin{array}{l}\text { Assessment of failures in the integration with hospital picture archive and communication system and electronic health } \\
\text { record systems, as well as to on-premise and public cloud services }\end{array}$ \\
\hline Malfunction & Occurrence of any fatigue of integrity or potential to induce to use errors \\
\hline Relevance & $\begin{array}{l}\text { Interviews to assess users' own judgement of helpfulness of the platform to guide them beyond obvious decisions for a } \\
\text { given data available set }\end{array}$ \\
\hline Added value & $\begin{array}{l}\text { Statistical assessment of occurrence of correct predictions for clinical end points that current diagnosis/prognosis } \\
\text { standard protocols could not predict correctly }\end{array}$ \\
\hline Usability/user friendliness & Observation of use patterns, users' eye tracking, interviews to users \\
\hline
\end{tabular}


European repositories for NB and DIPG. We aim to impact current clinical guidelines [40].

PRIMAGE is designed to have a major impact on improving the disease management of malignant solid tumours. Upon successful validation in NB and DIPG, evidencing how in silico tools can assist clinicians to make improved informed decisions, PRIMAGE platform's architecture, repository infrastructure, simulation frameworks, and web-based user interfaces will have the potential to be adapted and completed for use in the management of other types of cancer.

We do not foresee any intellectual property nor commercial issues during the project: the intellectual property background of each partner has been defined and declared already, as well as the general framework on the future exploitation and ownership of the project's results. At the end of the project, there will be pieces of software, computational models and other intellectual property assets that will be owned by the partner(s) that developed them. Exploitation and/or access rights on these developments may be given to the rest of partners under favourable conditions. This shall be set in specific commercial agreements between the owner(s) and the licensee(s) after the end of the project. Finally, regarding the access to the PRIMAGE platform as a whole, the aim of the project is to give open access to the research community. Means for the sustainability and continuity over time of the PRIMAGE platform will be assessed also during the project.

\section{Conclusion}

At the end of the project, the developed open cloudbased platform will support phenotyping (diagnosis), treatment stratification (prediction) and patient-specific CEPs determination (prognosis), based on the use of imaging biomarkers, tumour growth simulation, advanced visualisation of confidence scores, and machine learning approaches. The decision support prototype will be constructed and validated on NB and DIPG cancers. The results will be available for the scientific community and ready for transfer learning to other malignant solid tumours. Data infrastructures, imaging biomarkers, and predictive models for in silico medicine research will be validated during this project. Given the rarity of these tumours, international cooperative networking is essential to agglutinate relevant in silico data from clinical trials and large real-world data repositories, facilitating identification of effective clinical tools.

\footnotetext{
Abbreviations

API: Application programming interface; CEPs: Clinical endpoints; CPU: Central processing unit; DIPG: Diffuse intrinsic pontine glioma; DSS: Decision support system; HPC: High-performance computing; INRG: International neuroblastoma risk group; MEE: Model execution environment; NB: Neuroblastoma; PRIMAGE: Predictive in-silico multiscale analytics to support cancer personalised diagnosis and prognosis, empowered
}

by imaging biomarkers; QUIBIM: Quantitative Imaging Biomarkers in Medicine; SIOPE: European Society for Paediatric Oncology

\section{Acknowledgements}

PRIMAGE (PRedictive In-silico Multiscale Analytics to support cancer personalised diaGnosis and prognosis, empowered by imaging biomarkers) Business Place is a Horizon 2020 | RIA (Topic SC1-DTH-07-2018) project with grant agreement no: 826494.

\section{Authors' contributions}

$L M B, E N, A A B, I B, G M B$, and JMGA initially proposed to write the manuscript and defined the scheme of the content that was discussed with all authors. $\mathrm{LMB}, \mathrm{AC}, \mathrm{SG}, \mathrm{RL}, \mathrm{BH}$, and $\mathrm{BMH}$ edited the first version of the manuscript. Each author contributed to the intellectual content of the manuscript. In addition, AS, AAB, AJP, KS, AS, and MA prepared the figures. All authors read and approved the final version of the manuscript.

\section{Funding}

Horizon 2020 project (RIA, topic SC1-DTH-07-2018)

\section{Availability of data and materials}

Project website (https://www.primageproject.eu/)

Ethics approval and consent to participate

Not applicable.

\section{Consent for publication}

Not applicable.

\section{Competing interests}

The authors declare that they have no competing interests.

\section{Author details}

${ }^{1}$ Medical Imaging Department, La Fe University and Polytechnic Hospital \& Biomedical Imaging Research Group (GIBI230) at La Fe University and Polytechnic Hospital and Health Research Institute, Av. Fernando Abril Martorell 106, 46026 Valencia, Spain. ${ }^{2}$ Quantitative Imaging Biomarkers in Medicine, QUIBIM SL, Edificio Europa, Av. de Aragón, 30, Planta 12, 46021 Valencia, Spain. ${ }^{3}$ Children's Cancer Research Institute, Vienna, Austria. ${ }^{4}$ Instituto de Instrumentación para Imagen Molecular (I3M), Universitat Politècnica de València (UPV), $\backslash \backslash$ Camino de Vera s/n, 46022 Valencia, Spain. ${ }^{5}$ Biomedical Imaging Research Group (GIBI230), La Fe Health Research Institute, Av. Fernando Abril Martorell 106, Torre E, 46026 Valencia, Spain. ${ }^{6}$ Department of Pediatrics, Faculty of Medicine and University Hospital Cologne, University of Cologne, Cologne, Germany. ${ }^{7}$ Multiscale in Mechanical and Biological Engineering, Department of Mechanical Engineering, Universidad de Zaragoza, Zaragoza, Spain. ${ }^{8}$ Aragón Institute of Engineering Research, Zaragoza, Spain. ${ }^{9}$ Department of Computer Science, University of Konstanz, Konstanz, Germany. ${ }^{10}$ Medexprim, 815 La Pyrénéenne, 31670 Labège, France. ${ }^{11}$ Paediatric Oncology Unit, La Fe University and Polytechnic Hospital, Av. Fernando Abril Martorell 106, Torre G, 2 Floor, 46026 Valencia, Spain. ${ }^{12}$ European Society for Paediatric Oncology, Brussels, Belgium.

${ }^{13}$ Department of Computer Science and Insigneo Institute of In Silico Medicine, University of Sheffield, Regent Court, 211 Portobello, Sheffield, UK.

${ }^{14}$ Simulation, Modelling and Engineering Software, Ansys Group, Montigny-le-Bretonneux, France. ${ }^{15}$ ACC Cyfronet, AGH University of Science and Technology, Sano Centre for Computational Medicine, Nawojki 11, 30-950 Kraków, Poland. ${ }^{16}$ Chemotargets S.L., Carrer de Baldiri Reixac, 4-8 TI05A7 Torre I, planta 5, A7, 08028 Barcelona, Spain. ${ }^{17}$ Department of Industrial Engineering, Alma Mater Studiorum, University of Bologna, Bologna, Italy. ${ }^{18}$ Matical Innovation, Calle de Torija, 5, 28013 Madrid, Spain. ${ }^{19}$ Department of Translational Research, University of Pisa, Chair Radiodiagnostica 3, Pisa University Hospital, Via Roma 67, 56126 Pisa, Italy.

Received: 3 October 2019 Accepted: 24 February 2020

Published online: 03 April 2020

\section{References}

1. Porter ME, Teisberg EO (2006) Redefining health care : creating value-based competition on results. Harvard Business School Press, Boston 
2. Hernán MA, Robins JM (2016) Using big data to emulate a target trial when a randomized trial is not available. Am J Epidemiol 183:758-764. https://doi. org/10.1093/aje/kwv254

3. Wang SV, Schneeweiss S, Berger ML et al (2017) Reporting to improve reproducibility and facilitate validity assessment for healthcare database studies V1.0. Pharmacoepidemiol Drug Saf 26:1018-1032. https://doi.org/10. 1002/pds.4295

4. Viceconti M, Henney A, Morley-Fletcher E (2016) In silico clinical trials: how computer simulation will transform the biomedical industry. Int J Clin Trials 3:37. https://doi.org/10.18203/2349-3259.ijct20161408

5. Martí-Bonmatí L, Alberich-Bayarri A (eds) (2018) Imaging biomarkers : development and clinical integration. Springer, Heidelberg

6. Kazem MA (2017) Predictive models in cancer management: a guide for clinicians. Surgeon 15:93-97. https://doi.org/10.1016/j.surge.2016.06.002

7. Martí-Bonmatí L, Ruiz-Martínez E, Ten A, Alberich-Bayarri A (2018) How to integrate quantitative information into imaging reports for oncologic patients. Radiologia 60:43-52. https://doi.org/10.1016/j.rx.2018.02.005

8. Bernsen MR, Kooiman K, Segbers M, Van Leeuween FWB, Jong M (2015) Biomarkers in preclinical cancer imaging. Eur J Nucl Med Mol Imaging 42: 579-596. https://doi.org/10.1007/s00259-014-2980-7

9. Alberich-Bayarri A, Neri E, Martí-Bonmatí L (2019) Imaging biomarkers and imaging biobanks. In: Ranschaert E, Morozov S, Algra P (eds) Artificial intelligence in medical imaging. Springer, Heidelberg, pp 119-126

10. O'Connor JPB, Aboagye EO, Adams JE et al (2017) Imaging biomarker roadmap for cancer studies. Nat Rev Clin Oncol 14:169-186. https://doi.org/ 10.1038/nrclinonc.2016.162

11. Escribano J, Chen MB, Moeendarbary E et al (2019) Balance of mechanical forces drives endothelial gap formation and may facilitate cancer and immune-cell extravasation. PLoS Comput Biol 15:e1006395. https://doi.org/ 10.1371/journal.pcbi.1006395

12. Enderling $\mathrm{H}$, Chaplain MAJ (2014) Mathematical modeling of tumor growth and treatment. Curr Pharm Des 20:4934-4940. https://doi.org/10.1111/2041210X.12500

13. Bhatnagar SN (2012) An audit of malignant solid tumors in infants and neonates. J Neonatal Surg 1:5 PMID: 26023364; PMCID: PMC4420305

14. London WB, Castleberry RP, Matthay KK et al (2005) Evidence for an age cutoff greater than 365 days for neuroblastoma risk group stratification in the Children's Oncology Group. J Clin Oncol 23:6459-6465. https://doi.org/ 10.1200/JCO.2005.05.571

15. Cohn SL, Pearson ADJ, London WB et al (2009) The international neuroblastoma risk group (INRG) classification system: an INRG task force report. J Clin Oncol 27:289-297. https://doi.org/10.1200/JCO.2008.16.6785

16. Morgenstern DA, Pötschger $U$, Moreno $L$ et al (2018) Risk stratification of high-risk metastatic neuroblastoma: a report from the HR-NBL-1/SIOPEN study. Pediatr Blood Cancer 65:e27363. https://doi.org/10.1002/pbc.27363

17. Rubie H, De Bernardi B, Gerrard M et al (2011) Excellent outcome with reduced treatment in infants with nonmetastatic and unresectable neuroblastoma without MYCN amplification: results of the prospective INES 99.1. J Clin Oncol 29:449-455. https://doi.org/10.1200/JCO.2010.29.5196

18. De Bernardi B, Gerrard M, Boni L et al (2009) Excellent outcome with reduced treatment for infants with disseminated neuroblastoma without MYCN gene amplification. J Clin Oncol 27:1034-1040. https://doi.org/10. 1200/JCO.2008.17.5877

19. Kohler JA, Rubie H, Castel $V$ et al (2013) Treatment of children over the age of one year with unresectable localised neuroblastoma without MYCN amplification: results of the SIOPEN study. Eur J Cancer 49:3671-3679. https://doi.org/10.1016/j.ejca.2013.07.002

20. Canete A, Gerrard M, Rubie H et al (2009) Poor survival for infants with MYCNamplified metastatic neuroblastoma despite intensified treatment: the International Society of Paediatric Oncology European Neuroblastoma Experience. J Clin Oncol 27:1014-1019. https://doi.org/10.1200/JCO.2007.14.5839

21. Avanzini S, Pio L, Erminio G et al (2017) Image-defined risk factors in unresectable neuroblastoma: SIOPEN study on incidence, chemotherapyinduced variation, and impact on surgical outcomes. Pediatr Blood Cancer 64. https://doi.org/10.1002/pbc.26605

22. Monclair T, Mosseri V, Cecchetto G, De Bernardi B, Michon J, Holmes K (2015) Influence of image-defined risk factors on the outcome of patients with localised neuroblastoma. A report from the LNESG1 study of the European International Society of Paediatric Oncology Neuroblastoma Group. Pediatr Blood Cancer 62:1536-1542. https://doi.org/10.1002/pbc. 25460
23. Ladenstein R, Pötschger U, Pearson ADJ et al (2017) Busulfan and melphalan versus carboplatin, etoposide, and melphalan as high-dose chemotherapy for high-risk neuroblastoma (HR-NBL1/SIOPEN): an international, randomised, multi-arm, open-label, phase 3 trial. Lancet Oncol 18:500-514. https://doi.org/10.1016/S1470-2045(17)30070-0

24. Ladenstein R, Pötschger U, Valteau-Couanet D et al (2018) Interleukin 2 with anti-GD2 antibody ch14.18/CHO (dinutuximab beta) in patients with highrisk neuroblastoma (HR-NBL1/SIOPEN): a multicentre, randomised, phase 3 trial. Lancet Oncol 19:1617-1629. https://doi.org/10.1016/S14702045(18)30578-3

25. Johung TB, Monje M (2017) Diffuse intrinsic pontine glioma: new pathophysiological insights and emerging therapeutic targets. Curr Neuropharmacol 15:88-97. https://doi.org/10.2174/ $1570159 \times 14666160509123229$

26. Sacha D, Stoffel A, Stoffel F, Kwon BC, Ellis G, Keim DA (2014) Knowledge generation model for visual analytics. IEEE Trans Vis Comput Graph 20: 1604-1603. https://doi.org/10.1109/TVCG.2014.2346481

27. Rimrock. Robust Remote Process Controller. https://submit.plgrid.pl/. Accessed 28 Sept 2019

28. PL-Grid Data service. https://data.plgrid.pl/?locale=en. Accessed 28 Sept 2019

29. ATMOSPHERE Project. Adaptive, trustworthy, manageable, orchestrated, secure, privacy-assuring, hybrid ecosystem for resilient cloud computing ATMOSPHERE Project. https://cordis.europa.eu/project/rcn/211963/factsheet/ en. Accessed 8 Sept 2019

30. Model Execution Environment. https://mee.cyfronet.pl/. Accessed 30 Sept 2019

31. Kasztelnik M, Coto E, Bubak M et al (2017) Support for Taverna workflows in the VPH-Share cloud platform. Comput Methods Programs Biomed 146:3746. https://doi.org/10.1016/j.cmpb.2017.05.006

32. Nowakowski P, Bubak M, Bartyński T et al (2018) Cloud computing infrastructure for the VPH community. J Comput Sci 24:169-179. https://doi. org/10.1016/j.jocs.2017.06.012

33. Bubak M, Gubała T, Hose DR et al (2019) Processing complex medical workflows in the EurValve environment. Proceedings of the CompBioMed Conference, 25-27 September, 2019, London, UK. https://www. compbiomed-conference.org/wp-content/uploads/2019/07/CBMC19 paper_62.pdf

34. Martí Bonmatí L, Alberich-Bayarri A, García-Martí G et al (2012) Imaging biomarkers, quantitative imaging, and bioengineering. Radiologia 54:269278. https://doi.org/10.1016/j.rx.2010.12.013

35. Ingham-Dempster T, Walker DC, Corfe BM (2017) An agent-based model of anoikis in the colon crypt displays novel emergent behaviour consistent with biological observations. R Soc Open Sci 4:160858. https://doi.org/10. 1098/rsos. 160858

36. Richmond P, Walker D, Coakley S, Romano D (2010) High performance cellular level agent-based simulation with FLAME for the GPU. Brief Bioinform 11:334-347. https://doi.org/10.1093/bib/bbp073

37. An EC Research and Innovation Action. http://www.eurvalve.eu/. Accessed 28 Sept 2019

38. Weir P, Ellerweg R, Payne S et al (2018) Go-smart: open-ended, web-based modelling of minimally invasive cancer treatments via a clinical domain approach. arXiv:1803.09166. https://doi.org/10.13140/RG.2.2.30828.46726

39. GoSmart - Generic open-end simulation environment for minimaly invasive cancer treatment. https://gosmart-project.eu/. Accessed 28 Sept 2019

40. Simon T, Hero B, Schulte JH et al (2017) 2017 GPOH guidelines for diagnosis and treatment of patients with neuroblastic tumors. Klin Padiatr 229:147167. https://doi.org/10.1055/s-0043-103086

\section{Publisher's Note}

Springer Nature remains neutral with regard to jurisdictional claims in published maps and institutional affiliations. 